\title{
Response to "Letter to the Editor: Effects of Bariatric Surgery on Cancer Risk: Evidence from Meta-analysis"
}

\author{
Kui Zhang ${ }^{1} \cdot$ Yupeng Luo ${ }^{2} \cdot$ Hao Dai $^{1} \cdot$ Zhenhua Deng $^{1}$ \\ Published online: 13 February 2020 \\ (C) Springer Science+Business Media, LLC, part of Springer Nature 2020
}

\section{Dear editor,}

We read with interest "Letter to the editor: Effects of Bariatric Surgery on Cancer Risk: Evidence from Meta-analysis". Our meta-analysis [1] assessed the effect of bariatric surgery on overall cancer risk by comparing cancer risk in patients who had undergone bariatric surgery and cancer risk in patients with obesity who had not undergone weight reduction surgery. We evaluated the eligible studies if all the following conditions were met: [1] evaluation of the association of bariatric surgery and cancer risk; [2] inclusion of sufficient data or the data can be acquired to calculate ORs and $95 \%$ CIs; [3] the publication with the follow-up period at least 3 years; [4] all the studies were cohort studies; and [5] the study was published in English. Due to objective reasons, some valuable studies may be omitted because of insufficient data or data to calculate ORs and 95\% CIs.

Given the multiple mechanisms by which obesity increases the risk of cancer [2,3], we performed subgroup analysis; our results indicated that bariatric surgery was significantly associated with decreased breast cancer risk $(\mathrm{OR}=0.49,95 \%$ $\mathrm{CI}=0.33-0.72)$ and endometrial cancer risk $(\mathrm{OR}=0.43$, 95\% CI $=0.26-0.71)$. Reductions in cancer risk were strongest for postmenopausal breast and endometrial cancers. Both of these cancers are highly sensitive to estrogen levels $[4,5]$ and react rapidly to changes. Bariatric surgery results in rapid weight loss and has greater long-term success compared with non-surgical weight loss methods. Weight loss has been

Kui Zhang and Yupeng Luo contributed equally to this work.

Zhenhua Deng

newman-zhk@163.com

1 Department of Forensic Pathology, West China School of Basic Medical Sciences \& Forensic Medicine, Sichuan University, Chengdu 610041, Sichuan, People's Republic of China

2 West China Hospital, Sichuan University, Chengdu 610041, Sichuan, People's Republic of China shown to reduce levels of circulating estrogen [6], thereby decreasing the risk of these cancers.

However, due to the limitations of our study, further studies estimating the functional effect and side effects may eventually provide a better, comprehensive understanding.

\section{Compliance with Ethical Standards}

Conflict of Interest The authors declare that they have no conflict of interest.

\section{References}

1. Zhang K, Luo Y, Dai H, Deng Z. Effects of bariatric surgery on cancer risk: evidence from meta-analysis. Obes Surg 201921

2. Roberts DL, Dive C, Renehan AG. Biological mechanisms linking obesity and cancer risk: new perspectives. Annu Rev Med. 2010;61: 301-16.

3. Calle EE, Kaaks R. Overweight, obesity and cancer: epidemiological evidence and proposed mechanisms. Nat Rev Cancer. 2004;4(8): 579-91.

4. Key TJ, Appleby PN, Reeves GK, et al. Body mass index, serum sex hormones, and breast cancer risk in postmenopausal women. J Natl Cancer Inst. 2003;95(16):1218-26.

5. Kaaks R, Lukanova A, Kurzer MS. Obesity, endogenous hormones, and endometrial cancer risk: a synthetic review. Cancer Epidemiol Biomarkers Prev: a publication of the American Association for Cancer Research, cosponsored by the American Society of Preventive Oncology. 2002;11(12):1531-43.

6. de Waard F, Poortman J, de Pedro-Alvarez FM, et al. Weight reduction and oestrogen excretion in obese post-menopausal women. Maturitas. 1982;4(2):155-62.

Publisher's Note Springer Nature remains neutral with regard to jurisdictional claims in published maps and institutional affiliations. 